\title{
Impacto de los Procesos de Autoevaluación de los Programas de Especialidades Odontológicas de la Universidad de La Frontera - Chile
}

\author{
Impact to the Accreditation Processes of the Dental Specialty \\ Programmes of the La Frontera University-Chile
}

\author{
Ramón Fuentes Fernández ${ }^{1} \&$ Claudia Riquelme Macalusso²
}

\begin{abstract}
FUENTES, F. R. \& RIQUELME, M. C. Impacto de los procesos de autoevaluación de los programas de especialidades odontológicas de la Universidad de La Frontera - Chile. Int. J. Odontostomat., 15(2):342-347, 2021.

RESUMEN: La gestión por procesos en la educación superior se basa hoy en día en una visión de corte administrativo y de gestión, lo que conlleva a que en estas organizaciones se genere un cambio cultural profundo donde la mirada una gestión eficiente y eficaz transforme alas unidades educativas superiores de ser centros de generación de conocimiento académico a entes organizacionales que gestionan el conocimiento para la mejora de sus propios procesos. Así también, deben demostrar ser buenos no tan solo como entes formadores, sino que también organizaciones educativas que entregan un servicio de calidad, lo que ha implicado a la incorporación de procesos de autoevaluación como es el caso de las especialidades odontológicas de la Universidad de La Frontera para la mejora continua de sus procesos formativos de enseñanza-aprendizaje en el posgrado.
\end{abstract}

PALABRAS CLAVE: gestión del conocimiento, procesos de calidad.

\section{INTRODUCCIÓN}

En los distintos programas tanto en el pregrado como en el posgrado, la incorporación de los procesos de que se orientan a la implementación de sistemas de gestión de calidad, la Facultad de Odontología de la Universidad de La Frontera, en sus lineamientos estratégicos se centró desde el año 2013 a la consolidación de éste en sus distintos programas de pregrado como del posgrado. Es por ello que logra en el año 2015 la acreditación de las primeras especialidades odontológicas en Chile, lo que ha significado un logro relevante para la formación de especialistas en el sur de país. Este proceso exitoso se logró por la ejecución de manera sistemática de análisis, planeación y gestión estratégica para identificar en una primera fase las brechas que se debía mejorar sustancialmente en base a lo planteado por Aranda Gutiérrez (2005):
- Crear los lazos de confianza en la autogestión del quehacer de los programas.

- Mejorar la calidad en todas las áreas, procesos administrativos y programas académicos que se ofertan en la facultad.

- Mejorar y diversificar la oferta educativa de posgrado para hacerla más competitiva y pertinente.

- Incrementar la productividad de la generación del conocimiento, el desarrollo y transferencia tecnológica de los programas.

- Ordenar y reestablecer una mayor vinculación con sectores académicos específicos externos de cada uno de los contextos de los programas.

- Gestionar y administrar cuidadosamente los recursos financieros suficientes para atender las múltiples necesidades operativas de cada uno de los programas de especialidad, y

1 Dental School, Research Centre for Dental Sciences (CICO), Universidad de La Frontera, Temuco, Chile.

2 Dental School, Universidad de La Frontera, Temuco, Chile. 
- Convertir en cultura y práctica organizacional, los procesos de periódica, sistemática que implica el someterse a procesos de autoevaluación con fines de acreditación.

Con ello se logró construir una educación es de calidad, que en base a lo que plantea Alcántara Santuario (2007) permite que:

- Se pueda satisfacer las necesidades reales de cada una de las externalidades a los cuales están dirigidos los programas de especialidad en odontología (relevancia).

- Las oportunidades de formarse en programas de calidad hace que se logre una equidad en el acceso al perfeccionamiento profesional.

- El que se reconozca de manera externa la calidad formativa, significa que se alcanzan los objetivos propuestos por los programas, logrando la eficacia en su gestión, y

- Al tener claridad de cómo se deben optimizar los recursos, hace que los programas releven en la eficiencia en su propia gestión.

Por tanto, este cambio cultural organizacional al interior de la facultad de odontología de la universidad de La Frontera, y especialmente en los programas de especialidades formativas de posgrado, logra dirigirse a una gestión del conocimiento, que en términos de Álvarez Álvarez et al. (2013) los procesos de autoevaluación con fines de acreditación pueden:

- Crear conocimiento: Esto se logró través de la orientación, identificación y definición de las estratégicas implementadas en los planes de mejoramiento de los programas de especialidad odontológicas.

- Identificar conocimiento: En los procesos de diagnóstico de identificación de fortalezas y debilidades de los programas hizo que se redefinieran la misión y visión que tenían cada de uno de los programa, dándoles una mayor pertinencia de los mismos.

- Capturar conocimiento: Cada uno los mecanismos y fases de los procesos de autoevaluación hizo que los programas se adaptaran a como entenderán e incorporarían los datos de seguimiento de la progresión de los estudiantes, como insumo relevante para el control y aseguramiento de la calidad.

- Adaptar conocimiento: Con la nueva concepción de la importancia de los datos, estos fueron a su vez adaptados y adoptados por los programas para su propio beneficio en su gestión interna y externa de los programas.
- Organizar conocimiento: Una de las características muy características de los procesos de autoevaluación y en especial de los programas de especialidades odontológicas por su alto componente clínico es el manejo, gestión y administración de la información, sean evidencias o registros escritos del quehacer cotidiano del funcionamiento de estos.

- Almacenar conocimiento: En relación a lo anterior, estos procesos generaron la creación de repositorios digitales de la información para las nuevas acreditaciones, logrando así un acervo informático transversal para todos los programas de especialidad de la facultad.

Aplicar conocimiento: Uno de los principales resultados de los procesos de acreditación de las especialidades odontológicas fueron las mejoras en el desempeño organizacional desde la cotidianidad de la formación clínica como de la administración curricular (renovaciones y ajustes) de enfrentaron los programas de manera posterior a sus acreditaciones y producto de la reflexión permanente de sus procesos, ya que el cabio cultural trajo consigo cambios actitudinales en todos los programas que participaron, y por tanto, a los nuevos integrantes se realizan procesos de inducción en esta línea, ya que se instaló el sistema de gestión orientado a la calidad. Sin embargo, aún falta mejorar varios aspectos para lograr una mayor autonomía de los mismos programas para llevar a cabo estos procesos que son bastante agotadores para los equipo de trabajo.

\section{Principales hallazgos de los procesos de autoevaluación de especialidades odontológicas de la Facultad de Odontología de la Universidad de La Frontera}

El concepto de calidad tiene varias formas de entenderlo y comprenderlo, ya que según la mirada desde la gestión o desde la formación tendrá componente y elementos que guiarán su entendimiento aplicado, especialmente en las instituciones de educación superior. Ruiz (1999) en Alcántara Santuario presenta seis formas de observar la calidad como:

Producción original: Se asocia a instituciones o unidades de élite, cuya producción suele evaluarse de acuerdo con estándares internacionales.

- Resultado de la correlación entre metas, objetivos y resultados: Tiene relación con lo que se ofrece y lo que se produce, entre lo que se quiere y lo que se logra, estas instituciones por lo general están estrechamente vinculadas al mundo del trabajo.

- La mayor o menor distancia con respecto a un mo- 
delo que se considera como lo ideal o deseable para la institución o unidad académica: En este caso, la calidad consiste en el modelo debe definido por el la institución desde su propia planificación.

- Satisfacción de las necesidades del usuario: Tiene relación con la toma de opinión de estudiantes, empleadores, egresados, etc.

- Valor agregado: Suele entenderse por "valor agregado", a aquello que el estudiante aprende durante su permanencia en la institución.

- Un agregado de propiedades: Es el concepto de calidad que es más aplicable para aquellas actividades que originan productos tangibles, tales como publicaciones o patentes.

Así, las especialidades odontológicas, posterior a sus primeros procesos de autoevaluación entre los años 2014-2015 se generaron modificaciones internas profundas, no tan solo en lo académico sino que también en la gestión y administración de las mismas. La construcción de calidad y en función de los seis puntos, permite definir aspectos relevantes que posterior a las primeras acreditaciones de tres especialidades de la Facultad de Odontología, se entendería por calidad lo siguiente (Tabla I).

Sin embargo, para el logro exitoso del proceso de acreditación de especialidades odontológicas en una primera instancia, se hace fundamental considerar varios aspectos para la mejora interna de los procesos, no tan solo para las especialidades sino que para cualquier programa que se quiera acreditar. Es relevante a considerar los siguientes elementos:

A. Aspectos de planificación de los procesos. Realizar procesos de autoevaluación es formular planes de mejora o de desarrollo a cinco años ya que en términos de Boville et al. (2006).

Tabla I. Comparación entre las definiciones y los resultados de la construcción del concepto de Calidad de las Especialidades Odontológicas de la Universidad de La Frontera, Chile.

Producción original
Posterior a la acreditación y al convertirse la Facultad con el $100 \%$ de sus programas de espe cialidad acreditadas por la Comisión Nacional de Acreditación (CNA-Chile) conforma una construcción de formación de calidad no tan solo al mundo profesional de la odontología, sino que también será el Ministerio de Salud (MINSAL) quien lo reconocerá como referente nacional en la materia.

Resultado de I a correlación La idea original de avanzar y se $r$ los primeros en acreditar por la CNA-Chile era entre metas, objetivos y garantizar que la formación de e specialistas, en e ste caso en Ortodoncia, resultados Odontopeditría y en Trastornos Temporo Mandibulares y Dolor Orofacial (TTM y DOF), ya que a pesar que tener 10 años de existencia no existían los espacios tanto internos como externos para la certificación que diera cuenta del cumplimiento de los objetivos de cada uno de los programas. Mayor o menor distancia con
respecto a un modelo que se
considera como lo ideal o
deseable para la institución

Satisfacción de las necesidades del usuario

Valor agregado

El que todo proceso de autoevaluación esté acompañado y guiado no tan solo en lo me todológico sino que también en lo operativo del levantamiento de información para el logro de estos procesos, hace que la idea de calidad que tiene la institución no se pierda en el camino; al cumplir con roles de contraparte hace que se pueda cumplir con la idea de calidad que comparte la institución, en este caso la Universidad de La Frontera.

Este elemento de calidad, hizo que ahora todos los programas de especialidad odontológica releve y considere fundamental la participación de l os estudiantes, empleadores yt itulados en sus procesos no tan solo en la acreditación, sino que también en las renovaciones o ajustes curriculares llevados a cabo por los programas.

Al ser el valor agregado por antonomasia el logro del perfil de egreso, posterior a los procesos de acreditación, este elemento presente en los programas de asignatura, la difusión de los programas y en los reglamentos, pasa a ser el centro del cumplimiento de los objetivos y la demostración final de la calidad de la formación de especialistas en el área de la odontología.

Agregado de propiedades Finalmente, el comprender, aprehender, y aplicar la información cuantitativa del progreso de los estudiantes y como estos permiten el monitoreo permanente de los programas, hizo que las especialidades mejoraran sustancialmente aquello indicadores deficientes previos a los procesos de acreditación, lo que orienta ahora a un trabajo de mejora continua permanente, no tan solo a los programas acreditados, sino que aplica a las nuevas propuestas de programas de especialistas en algún área de la odontología. 
- Los hallazgos que se obtienen en las acreditaciones generan impactos en los equipos ya que asumen responsabilidades de mejora a través del control y seguimiento a través de la planificación.

- Los avances significativos de los programas hacen que se orienten a focalizarse de manera integral en sus propios procesos de mejora definidos en la planificación, y

- La misma consolidación de metas a corto y largo plazo está íntimamente relacionada con el sentido de pertenencia de sus miembros a esa organización.

Es por ello relevante para todo programa de especialidad odontológica el tiempo dedicado a la planificación y ejecución de acciones comprometidas por ellos mismos, ya que un componente importante en los procesos de re-acreditación es el cumplimiento de todos aquellos elementos deficientes de los programas identificados por ellos mismos o por la visita de los pares externos. Por eso es importante tener en consideración las siguientes fases que plantean García Naranjo \& Pino Batista (2013):

- Fase 1: Planificación de las acciones de la autoevaluación y sensibilización de los implicados.

- Fase 2: Determinación de los métodos de investigación a utilizar y elaboración de los instrumentos.

- Fase 3: Aplicación de los instrumentos y análisis e interpretación de los datos.

- Fase 4: Determinación de las fortalezas, debilidades y plan de mejora. Elaboración del informe.

B. Aspectos metodológicos del proceso. Una de las características de los programas de especialidades en odontología es su alto componente clínico curricular, lo que hace que el lenguaje que implica los procesos de autoevaluación sean complejos de incorporar y adoptar por los equipos de trabajo, prácticamente va paralelo al avance de los productos asociados a las acreditaciones de los programas. Es por ello necesario la incorporación de profesiones de otras áreas de la salud para lograr las adaptaciones comunicativas, comprensivas y de redacción de todo aquello que solicitan formalmente la CNA-Chile (Informe de Autoevaluación, Guía de Formularios y documentos anexos que evidencia lo declarado en ambos documentos). Es por ello que para Paredes Bodegas (2008) la "acreditación (quality assurance y validation) es un proceso complejo multifactorial de aseguramiento de la calidad de una institución o de un programa educativo que se basa en una evaluación previa de los mismos"; es por ello que estos procesos suponen esfuerzos para producir evidencias y productos aso- ciados que se validen como componentes de calidad en la formación de especialistas en odontología, para ello es importante siempre:

- Validar, examinar la planificación, las acciones y los logros propuestos a cinco años.

- Comprobar e interpretar las metas para la perfección de los programas.

- Incorporar la búsqueda de la mejora en la entrega de los aprendizaje a los estudiantes, y

- Buscar de manera permanente información válida para la toma de decisiones para la mejora de los mismos programas de especialización.

C. Aspectos administrativos del proceso. Todo proceso tiene una variable central, el tiempo, y para la mejora continua de los programas es aún más determinante el tener los tiempos definidos para el cumplimiento de las metas de mejora propuestos, ya que las reuniones de equipo, las reuniones con los estudiantes y titulados, las revisiones periódicas de los avances de los planes de mejora de manera semestral y anual y la implementación de seguimiento y monitoreo de satisfacción de los estudiantes de la formación entregada por sus profesores, etc. Significan esfuerzos mayores especialmente de los equipos de trabajo, ya que todas las actividades descritas van en un solo objetivo:

- Detectar fortalezas y debilidades, y posibilidades de mejoramiento.

- Diseñar planes de actuación para mejorar la calidad. - Proporcionar información sobre la calidad de los programas.

- Crear una cultura de calidad.

- Definir de forma específica el resultado que pretende conseguir.

- Identificar los elementos que necesita para obtener ese resultado final de la formación de especialidad.

- Reflexionar sobre todos estos elementos, de forma que permitan el resultado deseado en la planificación

Y al ser cíclico los procesos de autoevaluación donde las etapas de autoevaluación, evaluación externa y finalmente la acreditación, la institución debe entregar los lineamientos para la gestión de calidad, sin embargo, bajo la experiencia recogida en la Facultad de Odontología, el componente externo a la Facultad es solo un apoyo, pero no constituye una elemento clave en las acreditaciones de los programas de especialidad, ya que administrativamente, el que los programas de posgrado estén de manera dependiente fuera de la Facultad, aun cuando nacen y ope- 
ran en una facultad, hace que no exista un control más óptimo del funcionamiento de los programas de especialidad; esto ha conllevado que en los procesos de autoevaluación se agudice aún más esta brecha de eficiencia entre las unidades mayores y al facultad, donde los programas finalmente responden administrativamente a dos unidades y eso significa una duplicidad de los esfuerzos y un desgaste en la cotidianidad administrativa de los programas (López, 2002; Suárez Rosas et al., 2008; Ruiz-Quilcat \& Manrique-Chávez, 2016).

D. Aspectos económicos del proceso. El tiempo es una variable con un costo asociado directamente, por tanto a mayor tiempo planificado, mayores serán los costos de funcionamiento de los programas, y si no tienen una planificación financiera esto conlleva a inestabilidad en la sustentabilidad financiera. Así lo plantea (Andión-Gamboa, 2007), quien afirma que la "evaluación de los programas que se practica actualmente está por lo general ligada al acceso a recursos y eso se ha convertido en un problema". Por tanto, si la institucionalidad no está considerando en sus planificaciones los costos asociados a todas estas implementaciones de mecanismos de mejora continua generará que los programas a la larga no quieran participar o no tengan un espíritu de mejora, ya que las limitaciones para contratar mas horas de gestión o el financiamiento de profesionales de apoyo a estos procesos, hace que a futuro sea inestable la sustentación del sistema de aseguramiento de la calidad y lo más importante no genere retrasos en los nuevos procesos de autoevaluación con fines de acreditación desde el inicio de éste como en los tiempos de vigencia de la acreditación a través de los dictámenes de acreditación (Suárez Rosas et al., 2007; Vesga, 2013).

\section{E. Aspectos de interiorización académica de los} procesos. Realizar procesos de autoevaluación con fines de acreditación implica procesos de aprendizaje del mismo procedimiento de acreditación en sí, las normas que lo rigen, la organización a cargo y sus procedimientos internos de funcionamiento que tiene la misma CNA-Chile, hace que los equipos a cargo tengan que además de trabajar en sus procesos, estudiar y aprender nuevos conocimientos de gestión, administración y legalidad a la cual no están cercanos debido a que la formación de especialistas en un área específica de la odontología hace que sea una formación de posgrado netamente clínico-práctico. Para Catón y Vásquez (2010) estos factores "son dinámicos y cambiantes, estos factores pueden ser tangibles (interactividad) o intangibles (capital intelectual, o va- lor añadido de una red), dependiendo de lo que deseemos evaluar", por lo cual es fundamental que existan las instancias de formación en el área de acreditación y gestión basada en la calidad para estos equipo y quienes se integran de manera posterior a un proceso de autoevaluación, ya que los saberes aprendidos si hay continuidad se podrán realizar las mejoras de manera las eficiente y eficaz, en caso contrario, sí que perdida de capital humano capacitado atenta de igual forma a la sustentabilidad de los programas (Fresán Orozco, 2007; Mejías Acosta \& Cobo Muñoz, 2007; Quiroz, 2007; García Naranjo \& Pino Batista).

F. Aspectos normativos para los procesos. Una línea no menor que existe en los procesos de autoevaluación con los mecanismos de registro, ordenamiento y resguardo de la información, aun cuando va paralelo a la creación de los documentos exigidos por la ley (informe de autoevaluación y guía de formularios) son los anexos. Estos son pieza clave en la visita de pares y son el respaldo de la verificación de toda la información entregada en los documentos o solicitada por la comisión de pares durante su visita. Para los autores García Naranjo \& Pino Batista es imprescindible para una acreditación:

\footnotetext{
- Obtener toda evidencia documental posible.

- Determinar las debilidades o deficiencias a través de la documentación entregada, y

- La elaboración de un plan de mejoras por cada variable con sus verificadores identificados.
}

Para el logro positivo en este tema el manejo de las TIC's es el soporte documental relevante en la gestión de la misma información. Para Álvarez Álvarez et al. esto recobra importancia porque la gestión documental trae consigo:

- El permitir y facilitar la comunicación del sistema de gestión a todas las partes interesadas.

- Suministrar un marco de operación claro y eficiente, pues permite declarar la forma en que las actividades se llevan a cabo.

- Proveer evidencias objetivas de cómo lograr alcanzar los resultados y mejorar la eficacia y adecuación continua del sistema de gestión de la calidad.

\section{CONCLUSIONES}

Puede concluirse entonces, que dado su carácter multidimensional que significa la calidad en educación superior y especialmente en las especialidades 
odontológicas, alcanzar niveles óptimos de calidad en general, no es simple y requiere de poner en juego las voluntades y capacidades de los equipos de trabajo, contar con los recursos materiales suficientes y cuidar que los desequilibrios puedan ser resueltos con rapidez y eficacia. Todo ello demanda enormes y constantes esfuerzos de instituciones, y sobre todo los programas de especialidades odontológicas como fue el caso de la Facultad de Odontología.

FUENTES, F. R. \& RIQUELME, M. C. Impact to the accreditation processes of the dental specialty programmes of the La Frontera University. Int. J. Odontostomat., 15(2):342-347, 2021.

ABSTRACT: Process management in higher education today is based on a vision of administrative and managerial which leads to the creation in these organizations of a profound cultural change where the vision of an efficient and effective management transforms the higher educational units from being centers of generation of academic knowledge to organizational entities that manage the knowledge to improve their own processes. They must also prove to be good not only as training bodies, but also educational organisations that deliver a quality service, which has involved the incorporation of selfevaluation processes such as the dental specialties of the La Frontera University for the continuous improvement of its teaching-learning processes in the postgraduate.

processes.

KEY WORDS: knowledge management, quality

\section{REFERENCIAS BIBLIOGRÁFICAS}

Alcántara Santuario, A. Dimensiones de la calidad en educación superior. Reencuentro, 50:21-7, 2007.

Álvarez Álvarez, M. J.; Castro Rojas, M. \& Peña Guarín, G. Gestión del conocimiento: una perspectiva desde la gestión de la calidad y la gestión documental. SIGNOS, 5(2):101-18, 2013.

Andión-Gamboa, M. Sobre la calidad en la educación superior: una visión cualitativa. Reencuentro, 50:83-92, 2007.

Aranda Gutiérrez, H. Resultados obtenidos en la implementación de un sistema para la gestión de calidad en una institución de educación agrícola superior en México. Rev. Mex. Agronegocios, 9(7), 2005. Disponible en: https://www.redalyc.org/ articulo. oa?idp $=1 \&$ id $=14101710 \&$ cid $=99801$

Boville, B.; Argüello, N. \& Reyes, N. La acreditación como proceso dinamizador hacia la calidad. Actual. Investig. Educ., 68(1), 2006. Disponible en: https://www.redalyc.org/pdf/447/44760104.pdf

Fresán Orozco, M. Repensar la calidad en la educación superior en el nuevo milenio. Reencuentro, 50:52-9, 2007.

García Naranjo, M. A. \& Pino Batista, M. G. Sistema de evaluación y acreditación en la educación superior cubana: un modelo para la gestión de la calidad. Atenas, 1(21):34-53, 2013.
López, R. L. La gestión de la calidad en los servicios. Concienc. Tecnol., (19), 2002. Disponible: https://www.redalyc.org/pdf/944/ 94401905.pdf

Mejías Acosta, A. A. \& Cobo Muñoz, M. La autoevaluación: una iniciativa para la gestión de la calidad en educación superior. Ing. Ind., 28(3):35-9, 2007.

Paredes Bodegas, E. Acreditación universitaria: Garantía de Calidad en la educación superior?. Rev. Med. Herediana, 19(2):435, 2008.

Quiroz, E. Competencias profesionales y calidad en la educación superior. Reencuentro, 50:93-9, 2007.

Ruiz-Quilcat, C. \& Manrique-Chávez, J. E. Acreditación universitaria en las carreras de odontología en el Perú. Rev. Estomatol. Herediana, 26(2):85-91, 2016.

Suárez Rosas, L.; Barrios Osuna, I. \& González Espíndola, M. E. Sistema de evaluación y acreditación de carreras universitarias. Implantación en el Instituto Superior de ciencias Médicas de la Habana. Rev. Haban. Cienc. Med., 6(3), 2007. Disponible en: http://www.revhabanera.sld.cu/index.php/rhab/article/view/1333

Suárez Rosas, L.; Barrios Osuna, I. \& González Espíndola, M. E. Sistema de evaluación y acreditación para la carrera de estomatología. Rev. Haban. Cienc. Med., 7(2), 2008. Disponible en: http://scielo.sld.cu/scielo.php?script=sci_arttext\&pid=S1729519X2008000200015

Vesga, R. J. J. Cultura organizacional y sistemas de gestión de la calidad: una relación clave en la gestión de las instituciones de educación superior. Rev. Cient. Guillermo de Ockham, 11(2):89100. 2013

Dirección para correspondencia:

Prof. Dr. Ramón Fuentes Fernández

Research Centre for Dental Sciences

Dental School

Universidad de La Frontera

Av. Francisco Salazar 1145

Temuco

CHILE

E-mail: ramon.fuentes@ufrontera.cl 